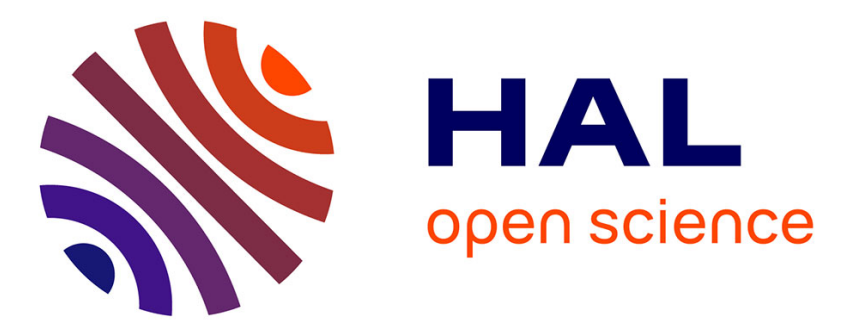

\title{
Mapping dynamical properties of cortical microcircuits using robotized TMS and EEG: Towards functional cytoarchitectonics
}

Sylvain Harquel, Thibault Bacle, Lysianne Beynel, Christian Marendaz, Alan Chauvin, Olivier David

\section{To cite this version:}

Sylvain Harquel, Thibault Bacle, Lysianne Beynel, Christian Marendaz, Alan Chauvin, et al.. Mapping dynamical properties of cortical microcircuits using robotized TMS and EEG: Towards functional cytoarchitectonics. NeuroImage, 2016, 10.1016/j.neuroimage.2016.05.009 . hal-01318810

\author{
HAL Id: hal-01318810 \\ https://hal.science/hal-01318810
}

Submitted on 14 Jun 2016

HAL is a multi-disciplinary open access archive for the deposit and dissemination of scientific research documents, whether they are published or not. The documents may come from teaching and research institutions in France or abroad, or from public or private research centers.
L'archive ouverte pluridisciplinaire HAL, est destinée au dépôt et à la diffusion de documents scientifiques de niveau recherche, publiés ou non, émanant des établissements d'enseignement et de recherche français ou étrangers, des laboratoires publics ou privés. 


\section{Mapping dynamical properties of cortical microcircuits using}

\section{robotized TMS and EEG: Towards functional cytoarchitectonics}

Sylvain Harquel ${ }^{a, b, c, d}$, Thibault Bacle ${ }^{a, b}$, Lysianne Beynel ${ }^{a, b}$, Christian Marendaz $^{a, b}$, Alan Chauvin ${ }^{a, b}$, Olivier David ${ }^{a, c, *}$

a Univ. Grenoble Alpes, F-38000 Grenoble, France

b. CNRS, UMR5105, Laboratoire Psychologie et NeuroCognition, LPNC, F-38000 Grenoble, France

c. Inserm, U1216, Grenoble Institut des Neurosciences, F-38000 Grenoble, France

d. CNRS, INSERM, Univ. Grenoble Alpes, CHU Grenoble, IRMaGe, F-38000 Grenoble, France

* Corresponding author:

Olivier David

Grenoble Institut des Neurosciences

Chemin Fortuné Ferrini - Bât EJ Safra - CHU

38700 La Tronche, France

Email: Olivier.David@inserm.fr

Tel: +33456520586

Fax: +33456520598 


\section{Abstract}

Brain dynamics at rest depend on the large-scale interactions between oscillating cortical microcircuits arranged into macrocolumns. Cytoarchitectonic studies have shown that the structure of those microcircuits differs between cortical regions, but very little is known about interregional differences of their intrinsic dynamics at a macro-scale in human. We developed here a new method aiming at mapping the dynamical properties of cortical microcircuits non-invasively using the coupling between robotized transcranial magnetic stimulation and electroencephalography. We recorded the responses evoked by the stimulation of 18 cortical targets largely covering the accessible neocortex in 22 healthy volunteers. Specific data processing methods were developed to map the local source activity of each cortical target, which showed inter-regional differences with very good interhemispheric reproducibility. Functional signatures of cortical microcircuits were further studied using spatio-temporal decomposition of local source activities in order to highlight principal brain modes. The identified brain modes revealed that cortical areas with similar intrinsic dynamical properties could be distributed either locally or not, with a spatial signature that was somewhat reminiscent of resting state networks. Our results provide the proof of concept of "functional cytoarchitectonics", that would guide the parcellation of the human cortex using not only its cytoarchitecture but also its intrinsic responses to local perturbations. This opens new avenues for brain modelling and physiopathology readouts.

\section{Keywords}

Transcranial magnetic stimulation; electroencephalography; cytoarchitectonics; functional neuroanatomy; cortical mapping 


\section{Introduction}

Brain dynamics at rest depend on the large scale interactions between local oscillators that correspond to cortical microcircuits arranged into macrocolumns (Jones, 2000). Cytoarchitectonic studies have shown that the structure of those microcircuits differs between cortical regions (Brodmann, 1909; Economo and Koskinas, 1925), but very little is known experimentally about interregional differences of their intrinsic dynamics at a macro-scale. In animal studies, there are some data relating specific cell types or processes to specific brain oscillations, e.g. giant pyramidal cells to beta oscillations in the motor cortex of the cat (Bouyer et al., 1987) or synaptic synchrony between excitatory pyramidal neurons and inhibitory interneurons to gamma oscillations in mouse entorhinal cortical slices (Salkoff et al., 2015). In humans, indirect evidence between brain dynamics and cytoarchitectonics from post-hoc correlation analysis of cortical maps has been suggested from analysis of resting state functional MRI spectral properties (Song et al., 2014). A significant association between cytoarchitectonic features of human cortical organization, in particular the size of layer 3 neurons, and whole-brain cortico-cortical connectivity has been recently identified, and suggests existing relationships between microscale cytoarchitectonics and macroscale connectomics (van den Heuvel et al., 2015). Because knowing how cytoarchitectonics influence brain dynamics is of outmost importance to increase our understanding of brain functioning, for example by developing more biologically grounded neuronal models in silico (Roy et al., 2014), we develop here a new experimental method using transcranial magnetic stimulation (TMS) to map the dynamical properties of human local cortical microcircuits non invasively. We mainly assume that the dynamical properties of neuronal responses to direct local perturbations depend on the way neurons are interconnected within cortical macrocolumns, i.e. local cytoarchitecture, and beyond, i.e. interregional laminar pattern of connections (Crick and Koch, 
1998). Operationally, we propose that spatio-temporal decoding of local responses to distributed focal brain stimulations may give rise to the possibility of studying functional cytoarchitectonics, that is the study of intrinsic dynamics of cortical microcircuits in vivo.

TMS consists of the generation of a transient magnetic field by a coil placed over the scalp, which induces electrical currents on the cortical surface (Wassermann et al., 2012) that activate responsive neurons (Mueller et al., 2014). Originally designed in order to better understand the actual effect of TMS and repetitive TMS on the ongoing neural activity, the coupling between TMS and electroencephalography (EEG) has recently given new insights regarding the nature and the properties of both the local neuronal microcircuits as well as their long-range connectivity (Bortoletto et al., 2015; Gosseries et al., 2015; Miniussi and Thut, 2010). These features can be assessed by the study of TMS evoked potentials (TEPs) (Casarotto et al., 2010; Ilmoniemi et al., 1997; Lioumis et al., 2009). TMS-EEG coupling also allows to study local and distributed brain oscillations at rest (Rosanova et al., 2009) or during sensorimotor (Fuggetta et al., 2005; Löfberg et al., 2013; Picazio et al., 2014) or cognitive tasks (Mattavelli et al., 2013; Rogasch and Fitzgerald, 2013; Vernet et al., 2015). TEPs were recorded over a large diversity of cortical areas including M1, frontal, parietal, and occipital lobes. As expected from the heterogeneity of cortical microcircuits and long range connectivity, TEPs showed distributed spatio-temporal patterns specific to each stimulated area, in terms of both spectral and spatial signatures (Cona et al., 2011; Rosanova et al., 2009; Thut et al., 2011). However, existing literature suffers from the diversity of the TMS parameters and of the EEG signal processing methods employed, which prevents any accurate understanding of the spatial organization of local cortical microcircuits' properties amongst cortical regions. 
In this study, we fully revisited the methods required to obtain an accurate mapping of dynamical properties of local microcircuits. Our methodology was based on the coupling of EEG recordings with neuronavigated and robotized TMS. Robotized TMS was critical because it allowed the automatic and precise positioning of the coil over a series of cortical targets (Ginhoux et al., 2013), thereby making possible the stimulation of a large number of cortical targets within a unique recording session. EEG processing methods were developed to estimate the early components of cortical current densities generating TEPs, locally for each cortical target, leading to a local source activity (LSA) map. Further spatial clustering on time-frequency properties of such local source activity was finally used to estimate the main cortical modes supposedly generated by the regional differences in cytoarchitecture and local microcircuitry.

\section{Materials and methods}

\subsection{Subjects}

This study was approved by the ethical committee of Grenoble University Hospital (ID RCB: 2013A01734-41), and registered on ClinicalTrials.gov (number NCT02168413). Twenty-two French native-speaker healthy volunteers (14 males, aged 29.6 \pm 10.1 years old) gave their written consent prior to the experiment and received payment for their participation. None of them had either history of psychiatric or neurological disorders, or history of alcohol or substance abuse. They were free of any medicinal treatment likely to modulate their cortical excitability levels. 


\subsection{Acquisition parameters}

\section{Protocol design}

MRI and TMS acquisitions were performed at IRMaGe MRI and Neurophysiology facilities (Grenoble, France). First, cerebral anatomical T1-weighted MRIs were acquired at 3T (Achieva 3.0T TX, Philips, Netherlands). The subjects were then prepared for the TMS-EEG experiment (EEG cap hanging), while their MRI were being processed in order to define the cortical targets. A co-registration step, necessary for the neuronavigation system, was performed. The resting motor threshold (rMT) was assessed during a classical motor CE session (see below). Finally, one TMSEEG mapping session per hemisphere was performed (30 min each), separated by a 15 min break. The order of the stimulated hemispheres was counterbalanced between subjects.

\section{TMS parameters}

Biphasic TMS pulses were delivered on a posterior to anterior direction using a Magpro Cool B65RO butterfly coil (MagVenture A/S, Denmark) plugged in a MagPro x100 TMS stimulator (MagVenture A/S, Denmark), and guided by a Localite neuronavigation system (Localite GmbH, Germany). The coil was handled automatically using a TMS-robot (Axilum Robotics, France). The rMT was assessed on the position eliciting the greatest motor evoked potential (MEP) on the contralateral first dorsal inter-osseous muscle. The MEPs were recorded using a Dantec Keypoint portable EMG recording system (Natus Medical Inc., USA). Using the threshold hunting method (Awiszus, 2003), the rMT was defined as the stimulation intensity that evoked a $50 \mu V$ MEP with a $50 \%$ probability. 
Both hemispheres were symmetrically stimulated. TMS-EEG mapping of a single hemisphere was performed in one session by recording the EEG activity evoked by the successive stimulation of 9 cortical targets defined in the standard Montreal Neurological Institute referential ([ $\pm \mathrm{x} \mathrm{y} \mathrm{z}]$ in $\mathrm{mm}$ ). The coil followed a predefined robotized sequence of stimulation, going from the anterior to the posterior parts of the brain (Figure 1): inferior frontal gyrus (IFG, [ $\left.\begin{array}{lll} \pm 6 & 24 & 13\end{array}\right]$ ), dorso lateral prefrontal cortex (DLPFC, [ \pm 424230$]$ ), middle frontal gyrus (MFG, [ \pm 341462$]$ ), anterior part of the supplementary motor area (SMA, [ $\pm 6 \quad 8$ 72]), primary motor cortex (M1, [ $\pm 36-3364])$, superior temporal gyrus (STG, [ $\pm 67-2310]$ ), superior parietal lobule (SPL, [ $\pm 8-6270]$ ), inferior parietal lobule (IPL, [ \pm 53 -52 51]), and superior occipital lobe (SOL, [ $\pm 25-87$ 33]). The target coordinates were projected back on each subject's individual anatomy using the inverse spatial transform given by the MRI normalization procedure of SPM8 software (www.fil.ion.ucl.ac.uk/spm8). The coil was placed on a posterior to anterior direction, with an angle of $45^{\circ}$ to the nasion-inion axis, except for M1 (perpendicular to the primary motor gyrus), SMA $\left(5^{\circ}\right), \mathrm{STG}$ and SOL (perpendicular to the axial plane). These angles were used according to the mechanical constrains introduced by robot's motion, while keeping standard coil orientations used in the literature if possible (Janssen et al., 2015). The sham condition consisted of stimulating 3 to $5 \mathrm{~cm}$ above one of the cortical targets (randomly distributed between subjects) at the highest intensity used within each subject (see below), in order to produce a click sound of maximum intensity. Each cortical target was stimulated during $2 \min 30 \mathrm{~s}$ at instantaneous frequency around $0.5-0.7 \mathrm{~Hz}$ in a random manner, so that no phase effect could build up as demonstrated by others on corticospinal excitability (van de Ruit et al., 2015). This led to an average number of 90 trials per stimulation point. The intensity was fixed at $120 \%$ of rMT, and adjusted according to the scalp-cortex distance measured from subject's anatomical MRI using the Stokes formula (Stokes et al., 2007; 2005). During each stimulation 
sequence, the subject was told to relax (resting state) and to stare at a black cross located on the opposite wall. Subjects were also listening to white noise through active noise cancellation intraauricular earphones (Bose QC 20, USA) in order to limit the influence of the auditory processing of the TMS click on the ongoing EEG activity. The sound level was adjusted individually to each subject, until the TMS click delivered at $95 \%$ of the stimulator output became barely audible. A thin layer of soft plastic was placed on the coil surface in order to limit both sensory and auditory feedbacks to the subject.

\section{EEG acquisition}

EEG was recorded using a 64 channels TMS compatible system (BrainAmp DC amplifiers and BrainCap EEG cap, Brain Products GmbH, Germany). The EEG cap was placed at the beginning of the experiment following the 10-20 standard system. Electrooculogram of the right eye was recorded using one of the 64 electrodes. The electrode impedances were adjusted and kept under 5 kOhms using conduction gel. The impedance levels were checked and corrected if needed before the two TMS-EEG sessions. The signal was recorded using the amplifier in DC mode, filtered using a $500 \mathrm{~Hz}$ anti-aliasing low-pass filter, and finally digitized at $1 \mathrm{kHz}$ sampling frequency. Electrode Fz was used as reference during the recording. The channel coordinates relative to the subject's scalp were measured at the end of the experiment using the neuronavigation system. 


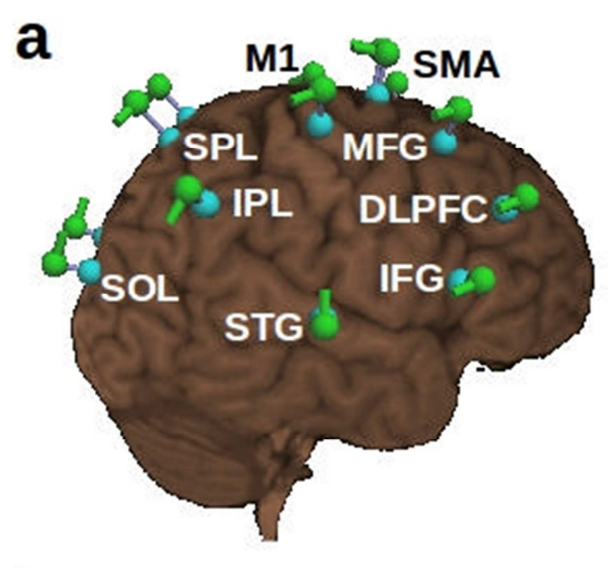

b

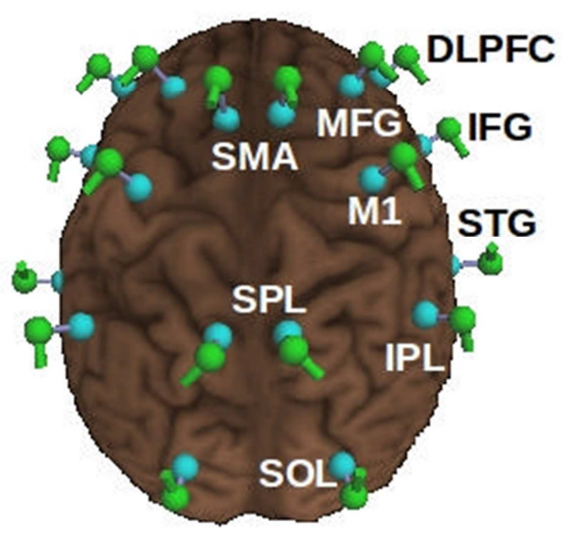

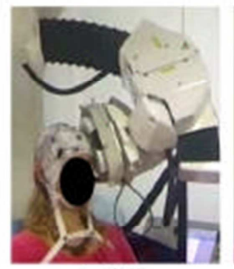

IFG

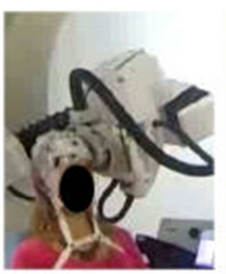

STG

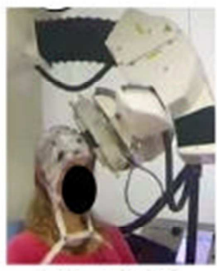

DLPFC

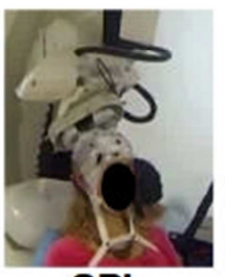

SPL

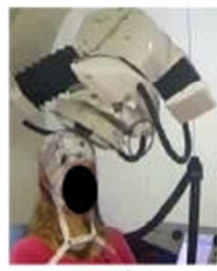

MFG

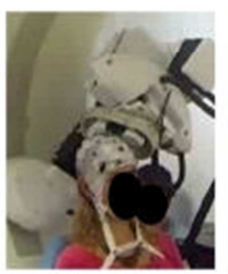

IPL

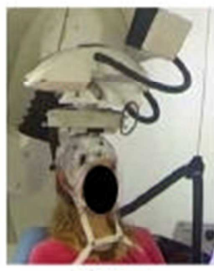

SMA

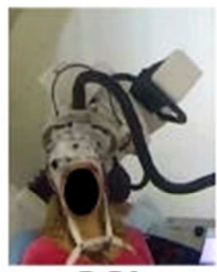

SOL

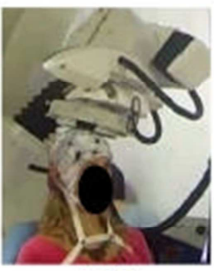

M1

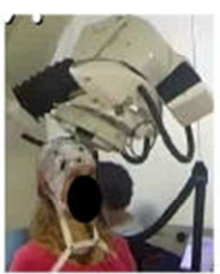

Sham

Figure 1: Cortical mapping procedure. A: Cortical targets used for the mapping procedure. Entry points and coil orientations are shown in green; cortical targets are in blue. Targets are symmetrically distributed on both hemispheres. B: Robotized sequence of stimulations. The snapshots show the position of the robotized arm throughout the mapping procedure of the left hemisphere, from the anterior to the posterior sites. Snapshots are sorted into time order from top left to bottom right, except for Sham condition performed above M1 in this example (see main text).

\subsection{EEG processing}

EEG signals were processed using Fieldtrip (Oostenveld et al., 2011) and Brainstorm 3 (Tadel et al., 2011) softwares, and other custom scripts written in Matlab (The MathWorks Inc., USA). 


\section{Preprocessing}

EEG signals were pre-processed semi-automatically based on the methodology described in (Rogasch et al., 2014), for each condition (18 targets and 1 sham) and each subject. First, the channels showing electrical noise (flat signal or peak-to-peak amplitude superior to $100 \mu V$ ) spanning more than $15 \%$ of the trials were discarded from the analysis (on average, $1.53 \pm 2.08$ channels per condition). EEG signals were then epoched around the TMS pulse, using a -200 to $+1000 \mathrm{~ms}$ time window of interest. TMS artifacts were discarded by cutting out the -5 to $+15 \mathrm{~ms}$ period surrounding the TMS pulses. Two rounds of independent component analysis (ICA) were then applied in order to remove noise remaining in the signal. The first ICA suppressed the muscle artifacts, while the second aimed at removing the decay artifact, ocular activity, auditory-evoked potentials and other noise-related artifacts (Rogasch et al., 2014). Before the second ICA, the signal was spline interpolated over the -5 to $+15 \mathrm{~ms}$ period, band-pass filtered $(1-80 \mathrm{~Hz})$, re-referenced using the average reference, and cleaned from bad trials (leading to a mean of $77.7 \pm 7.2$ trials left per condition). The ocular components were automatically identified using a threshold of 0.7 on the correlation product $\rho$ between the spatial topographies of the components and a template of typical horizontal eye movements and blinks build from our own database by averaging over subjects. Other artifact components (decay, auditory-evoked potentials and other noises) were detected by thresholding the z-score (above 4) of their mean activity against the pre-stimulus period, and by visual inspection. On average, 19.2 (+/- 7.7) components were removed from the signal. Cleaned EEG time series were reconstructed using the remaining components and any isolated channel still showing remaining noise was discarded from further analysis. Time series of rejected channels were finally inferred using the activity averaged over their neighboring channels. 


\section{TMS evoked potentials (TEPs)}

TEPs were computed for each condition and subject by averaging over trials, using a baseline normalization (z-scoring) over the -200 to $-5 \mathrm{~ms}$ period. The grand average TEP was obtained for each TMS target by averaging across subjects.

\section{TEP source reconstruction}

TEP source reconstruction was performed following the default procedure proposed in Brainstorm 3 software (Tadel et al., 2011). First, the cortex and head meshes (15000 and 10000 vertices respectively) of each individual were generated using the automated MRI segmentation routine of FreeSurfer (Reuter et al., 2012). The locations of EEG electrodes were co-registered on each subject's anatomical MRI. The forward model was then computed using the symmetric Boundary Element Method developed in the openMEEG freeware, using default values for conductivity and layer thickness (Gramfort et al., 2010). The full noise covariance matrix was then computed for each subject using the temporal concatenation of the baseline periods of all conditions. Sources were distributed orthogonally to the cortical surface and their amplitudes were estimated using the default values of the Brainstorm implementation of the whitened and depth-weighted linear L2minimum norm solution. The source amplitudes were finally normalized (z-score against prestimulation baseline). 


\section{Local source activity map}

The LSA map was constructed in order to represent the cortical response to TMS within the region of interest (ROI) centred on each target, for all the 18 stimulated sites simultaneously (Figure 2). ROIs were created on each individual anatomy using a mean spatial extent of $10 \mathrm{~cm}^{2}$, covering about 50-60 vertices of cortical mesh. The LSA time series of ROI $i$ and subject $k, S_{i}^{k}$, was obtained by extracting the first mode of the principal component analysis decomposition of the source time series evoked by the stimulation of the corresponding stimulation target over all the vertices within ROI $i$. This corresponds to a single LSA time series per ROI. For display purposes, the LSA map was created by projecting back the group average (grand average of absolute values) of LSA time series of each ROI on a canonical brain (using Colin27 template). LSA values were interpolated in between ROIs using the Shepard's weighting of 3D nearest-neighbor interpolation in order to assign a LSA value to every node of the cortical mesh. 


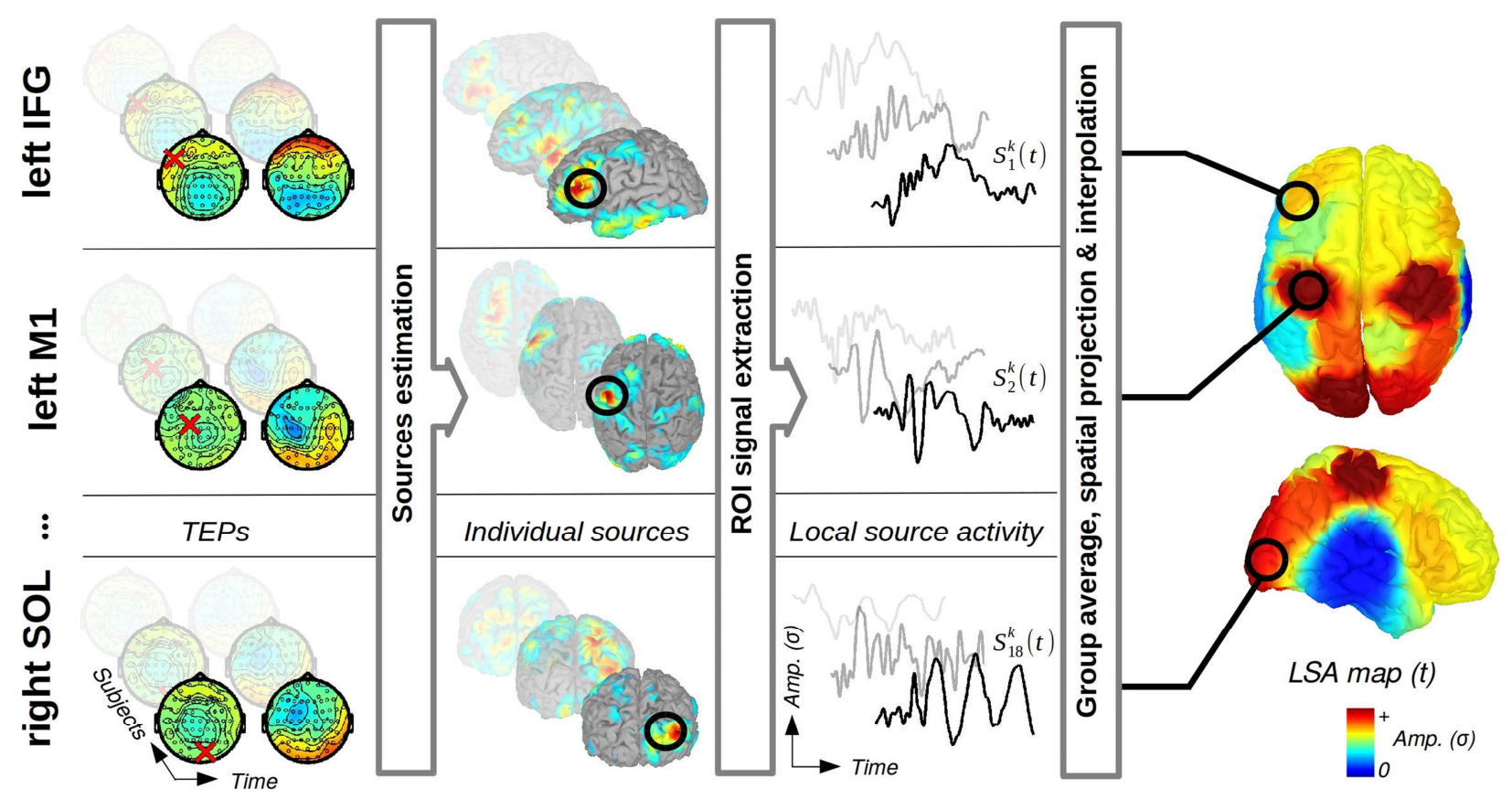

Figure 2: Generation of the LSA map. Sources of TEPs to each stimulated region (red cross) are first estimated. Local cortical responses (LSA time series $S_{i}^{k}$ ) are then inferred from source time series averaged from ROIs centered on each cortical target (black circles). The LSA map is finally generated using spatial interpolation of the group average (grand average of absolute values) of LSA time series.

\section{LSA mode analysis}

In order to attempt to connect LSA responses to underlying cytoarchitectonic properties through the identification of brain modes, we proceeded to a group ICA analysis. We assumed here that the neuronal populations participating to the same modes show the same dynamical signatures because of shared cytoarchitectonics. The group ICA was performed on the LSA time series $S_{i}^{k}$ of each ROI $i$ and subject $k$ on the TEP period of interest (from -50 to $+400 \mathrm{~ms}$ ). Here, we assumed no interhemispheric differences, and thus considered the left and right LSA responses as a repeated measurement of the same process. The LSA group (over subjects and hemispheres) ICA was 
performed after the concatenation of LSA matrices along the temporal dimension (Calhoun et al., 2009), leading to a group LSA matrix $M_{i}=\left[S_{i}{ }^{1} \ldots S_{i}{ }^{k} \ldots S_{i}{ }^{K}\right]$ of size $[L / 22 N K]$, where $L$ is the number of targets (18), $N$ the number of time bins and $K$ the number of subjects (22). The matrix $M_{i}$ was thus decomposed into $L / 2$ (9) independent components (data dimension) using the logistic infomax ICA algorithm (Bell and Sejnowski, 1995) with the natural gradient feature from Amari, Cichocki \& Yang (Makeig et al., 1996). The dynamical signature of each component in each individual was finally assessed by means of its time/frequency (TF) decomposition obtained using Morlet wavelet transform between 9 and $50 \mathrm{~Hz}$ (window width of 7 cycles, $0.5 \mathrm{~Hz}$ bandwidth). Individual TF maps were normalized (z-score against baseline) and averaged across subjects. The output of the LSA mode analysis results are maps showing ROIs sharing common neuronal signatures, as exemplified by their time frequency decomposition.

\section{Statistics}

Significant spatial differences in the LSA map across stimulated areas were assessed over time using the Skillings-Mack test (Chatfield and Mander, 2009). This test is derived from the Friedman test (non-parametric equivalent of the repeated measures ANOVA test) and can handle missing data. Because the same test was run at every time sample, p-values were temporally corrected: Differences were considered as significant at $p<0.05$ for at least 20 consecutive time bins (Blair and Karniski, 1993; Carota et al., 2010). The between subjects variability and the reproducibility of the LSA map was estimated over time by computing Spearman correlations between the LSA map obtained with all the 22 subjects, and LSA maps obtained with random subsets of subjects. 100 maps were computed for each time sample and each random subsets containing from 5 to 20 subjects. Statistical dependences between LSA and other experimental values were computed using Spearman correlations. Statistical significance of TF maps of ICA components was obtained using 
paired comparisons against baseline. A non-parametric Wilcoxon test was performed per timefrequency bin, and the resulting p-values were spatio-temporally corrected. Differences were considered as significant at $\mathrm{p}<0.05$ for at least 20 consecutive time bins and 3 adjacent frequencies (tiles of $20 \mathrm{~ms} \times 1.5 \mathrm{~Hz}$ ).

\section{Results}

Most of the subjects went through the entire mapping procedure without any major issue at an intensity of $120 \%$ of rMT. However, three subjects reported painful sensations during the stimulation of IFG and STG. Stimulation intensity was then lowered down (minimum of 105\% of rMT) for those three subjects when targeting IFG and STG. The TMS pulses still remained painful for one subject thus the experiment was stopped. In addition, one subject experienced drowsiness during the stimulation of some targets, which made the corresponding data not useable. In summary, we did not process the data of these four subjects for left IFG, of two subjects for left and right STG, and of one subject for left and right IPL, left and right SOL, right IFG, right DLPFC, right SMA, and right M1.

\subsection{TMS evoked potentials}

Figure 3 summarizes the different preprocessing steps used to estimate the TEPs. Figure 4 shows grand-average TEPs for the stimulation of the left hemisphere, the TEPs corresponding to the right hemisphere being very similar though mirrored along the interhemispheric axis. The sham condition did not show any significant activity, which suggests that the responses observed for real 
stimulations were not due to confounding auditory responses to TMS clicks. The earliest components $(<60 \mathrm{~ms})$ were rather focal under the target, indicating the activation of local neuronal populations. The largest activity within this period was generated by the stimulation of M1 (P30 component). In opposite, IPL was the least activated area, showing no activity pattern above baseline level. Larger amplitude responses could be observed after $60 \mathrm{~ms}$ for each condition, peaking around 100, 200 and $280 \mathrm{~ms}$. Whereas the 100 and $280 \mathrm{~ms}$ components were essentially generated after the stimulation of MFG and parieto-occipital areas, and of IFG, DLPFC and STG respectively, the $200 \mathrm{~ms}$ central component could be observed in nearly all the conditions. Although being potentially contaminated with auditory or somatosensory confounds (see Discussion below), these late components could reveal the level of efferent connectivity of each stimulated area. In particular, the stimulation of the DLPFC, IFG and STG, which are three cortical areas highly involved in inhibitory circuits and language processing respectively, generated the largest and longest activity (up to $340 \mathrm{~ms}$ ). In contrast, M1 stimulation led to the smallest late response, possibly due to the lesser proportion of cortico-cortical efferents. 


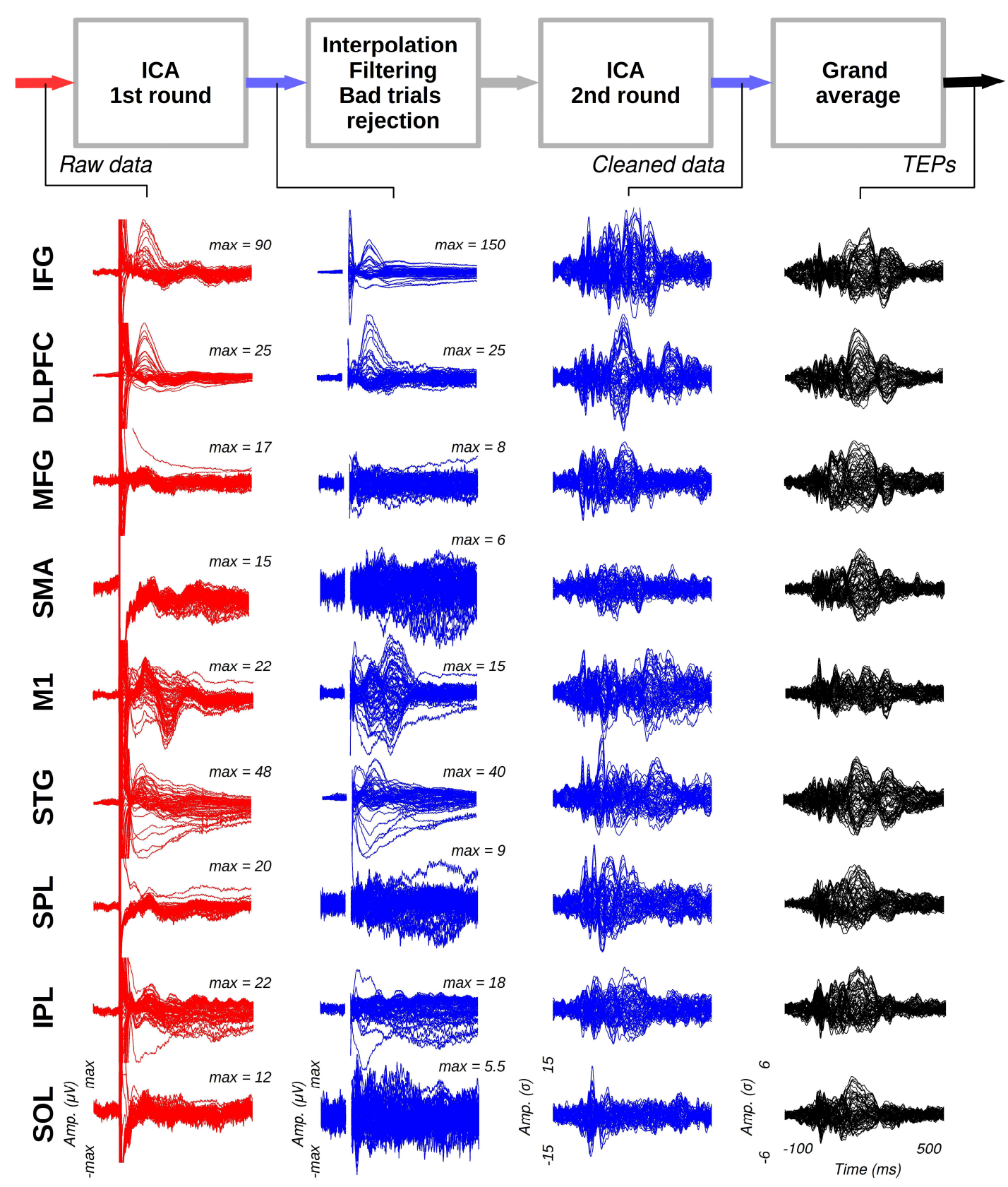

Figure 3: EEG preprocessing steps of the procedure proposed in (Rogasch et al., 2014). Signals are displayed using a butterfly view for all channels and for all left stimulation sites. Raw (red) and cleaned (blue) data come from a typical subject. Grand average TEPs (black) correspond to the average of TEPs across subjects. Note that the amplitude scale is varying between the two first columns to optimize the display. 


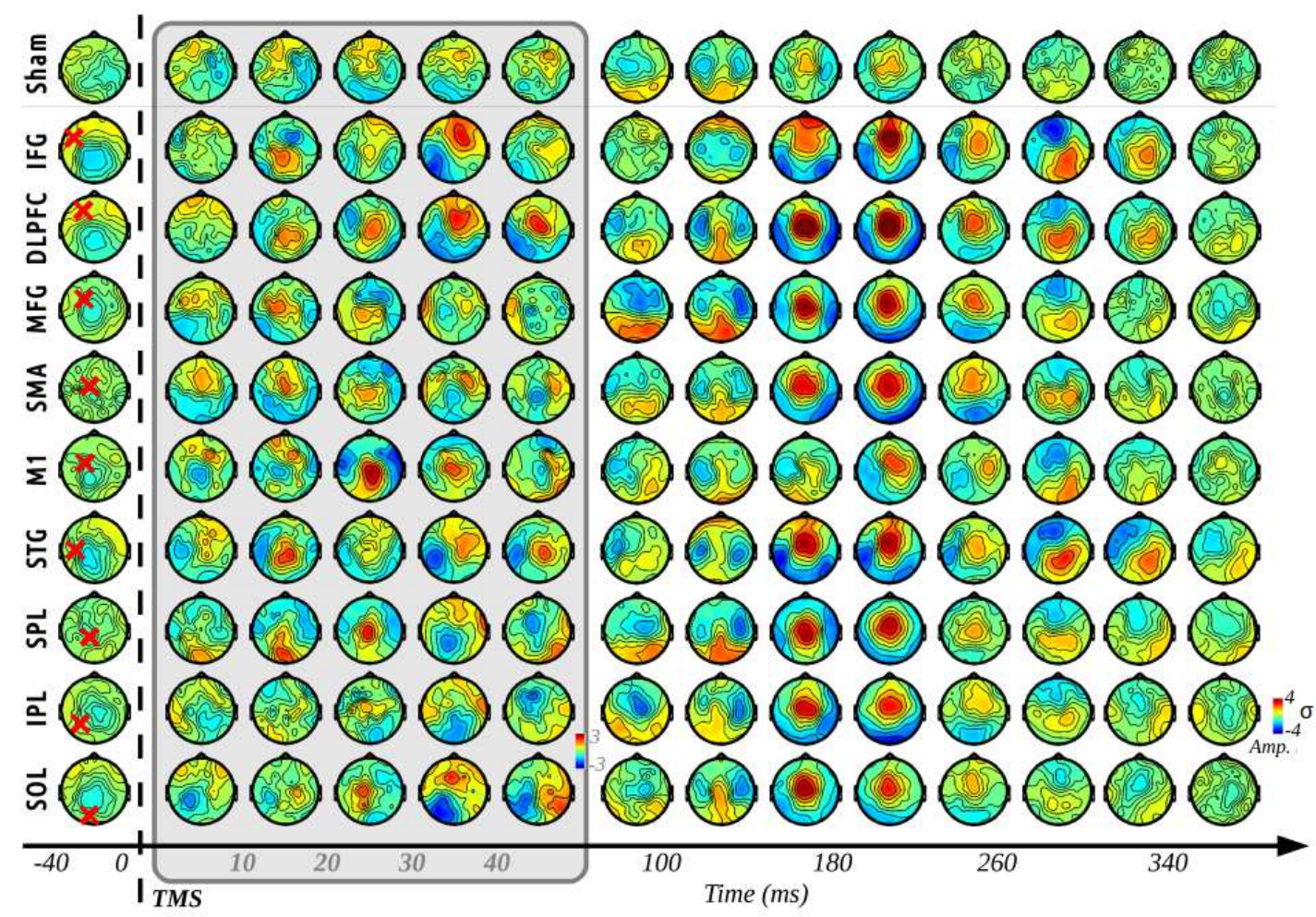

Figure 4: Grand-average TEPs for the stimulation of the left hemisphere. The grey insert emphasizes the earliest components. The z-score colorbar limits are -3 to +3 within this period. Topographies were obtained by averaging TEPs within a $40 \mathrm{~ms}$ time window (10 ms in grey insert). Red crosses on the left column indicate the stimulation sites.

\subsection{Local source activity}

Figure 5 shows the group average of LSA time series, i.e. the local cortical response to TMS within all stimulated areas. In most ROIs, LSA went back to baseline after $250 \mathrm{~ms}$ and reached its maximum amplitude in the earliest components (Figure 5A). Each ROI had its own temporal pattern of response to TMS that differed significantly from sham condition. The local response differed significantly across the 18 sites within all the period of interest [-50, $400 \mathrm{~ms}$, except for 3 
periods ranging from 49 to $53 \mathrm{~ms}$, from 104 to $121 \mathrm{~ms}$, and from 164 to $174 \mathrm{~ms}$. Besides, the LSA map appeared to be symmetric during all the analysis window (Figure 5B), as no significant difference between right and left hemispheres activation levels could be found. The only significant asymmetry could be observed in SOL around $70 \mathrm{~ms}$. M1 and SOL were the two first areas to respond at $30 \mathrm{~ms}$, followed by SMA and MFG at 45 and $70 \mathrm{~ms}$, the frontal and temporal areas (IFG, DLPFC, and STG) being activated later. The IPL was the least activated area throughout all the period of interest. Finally, the LSA map appeared to be highly reproducible within all the period of interest for groups of at least 18 subjects $(\rho=0.90+/-0.04)$, while a good correlation score $(\rho=$ $0.75+/-0.08$ ) can still be achieved for groups of at least 12 subjects. The reproducibility however varied across time bins, two of the three non-significant periods (from 104 to $121 \mathrm{~ms}$, and from 164 to $174 \mathrm{~ms})$ being also the most variable and least reproducible periods $(\rho=0.77+/-0.12$ and $\rho=$ $0.55+/-0.18$ for group of 18 and 12 subjects respectively). 


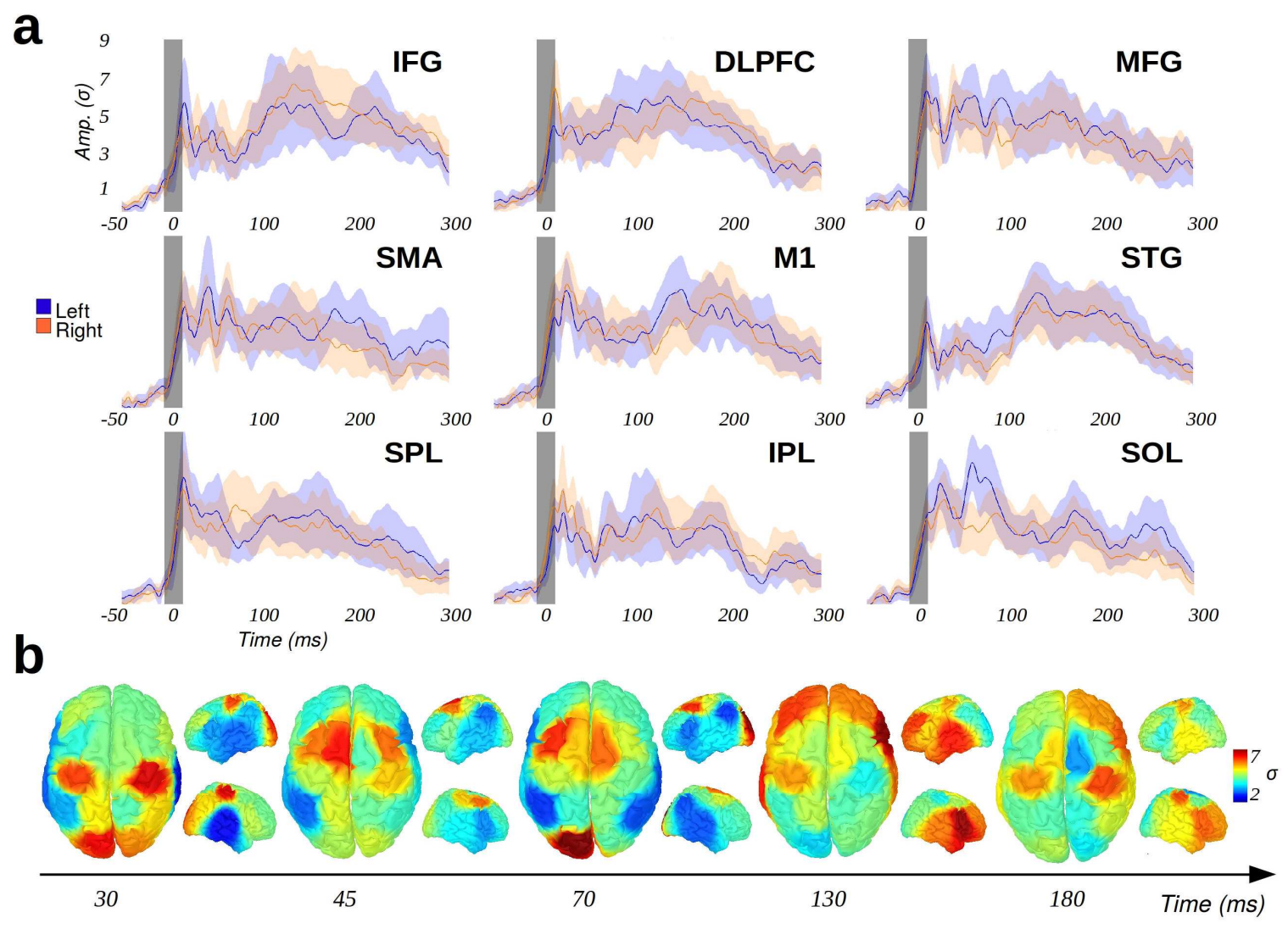

Figure 5: Group average of LSA time series. A: Group average (grand average of absolute values) of LSA time series for each region of interest. Colored areas represent the 95\% confidence intervals. The grey bar indicates the -5 to +15 ms interpolation period due to the TMS artefact. B: LSA map generated for the 5 time periods presenting the highest overall activity.

A group ICA on LSA time series was performed in order to detect cortical regions sharing common neuronal signatures, i.e. cortical modes (Figure 6). The temporal signature of each component was decomposed in the time-frequency domain using a wavelet transform in order to indicate their main frequencies. Interestingly, we could identify networks composed of several cortical areas for the majority of the components. Different fronto-parietal networks composed the components \#3, 8 and 9, while a temporo-parietal cluster was found in component \#1. The other components showed other networks mainly weighted by one area (component \#2: DLPFC; component \#4: IFG; component \#5: M1; component \#6: SOL), and component \#7 was only composed of the SPL. Each component had a specific dynamical signature, showing a mixture of responses in the low and high frequency 
bands. The most powerful and sustained alpha rhythm activation was found in component \#6 involving the occipital lobe (9-12 Hz, from 50 to $500 \mathrm{~ms}$ after stimulation onset). Alpha oscillations were also prominent in components \#1, 2, 8 and 9, all involving occipital or parietal areas in various proportions. Networks composed of motor areas (M1 and SMA) presented beta oscillations between 15 and $30 \mathrm{~Hz}$ at the 100-250 ms latency (components \#3, 5 and 8). Finally, low gamma oscillations (30-50 Hz) could be found around $100 \mathrm{~ms}$ after stimulation onset on several components (\#1, 2, 69). The highest frequencies were found in components \#2 and 9, mainly representing frontal areas (DLPFC and MFG respectively).
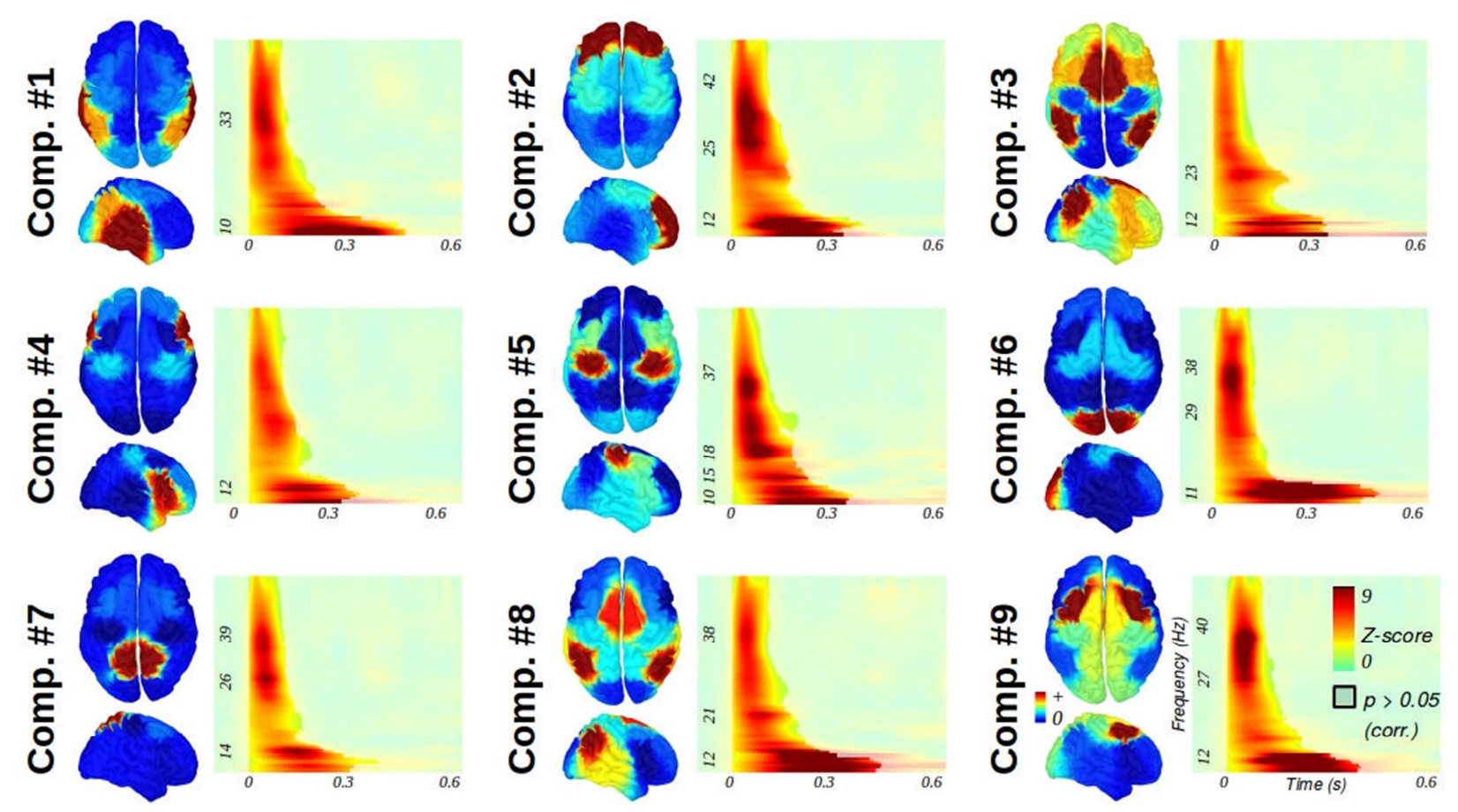

Figure 6: LSA modes identified from the components of a group ICA. Components were sorted by the amount of explained variance in descending order. Their topography is shown on the left, and their time-frequency profile on the right. Frequency bands corresponding to local maxima of power are indicated on the y axis. 


\section{Discussion}

We developed here the first attempt to broadly map the intrinsic dynamical properties of human cortical microcircuits. Our results are promising because homologous regions shared common properties and distinct neurodynamical responses were obtained in different regions, in particular along the anterior-posterior axis regarding high EEG frequencies. Under the assumption that impulse responses of local microcircuits are a proxy of their underlying cytoarchitecture, we suggest that our study paves the way to functional cytoarchitectonics, where one could envisage to proceed to the functional parcellation of the human brain using active probing of intrinsic cortical dynamics. Though our approach still suffers from technical limitations discussed below, and will remain limited in healthy subjects to the superficial cerebral cortex, it complements current approaches based on structural and resting state functional MRI because of its different neurodynamical content.

\subsection{Limitations of the study}

Nowadays, there is still a lack of fully data-driven and automatic pipelines for processing EEG data recorded during TMS. In this study, we used one of the latest methodology described in the literature. Rogasch et al. (2014) recommended the use of two rounds of ICA in order to filter out artifactual signals. Even if we developed some functions for automatically choosing the components to be rejected, there is still a need for a visual inspection of each of the remaining components. This step has to be eliminated in the future because it is time-consuming and introduces expertdependent variability. 
Auditory and somatosensory evoked potentials due to the sensory feedback of the scalp muscle contractions might also have partly influenced the recorded EEG activity. In principle, the TEP component at $200 \mathrm{~ms}$ could reflect the presence of such unwanted responses, regarding its overall latency and topography (Rogasch et al., 2014). However, several strong arguments are in favor of their minimized influence. First, from results not shown in this report, we did not find any correlation between the intensity of stimulation, which is directly linked to the power of both the click sound and the induced muscle twitch, and the amplitude of this component, which should be large in case of purely auditory or sensorimotor artifacts (Hegerl and Juckel, 1993). Second, this component has already been found and discussed in numerous studies in TMS-EEG (Braack et al., 2015; Chung et al., 2015). It has been shown to reflect a consistent aftereffect of the stimulation rather than a pure auditory or somatosensory artifact. Finally, such components have also been found in intracranial cortico-cortical evoked potentials after direct electrical stimulation of various areas (Keller et al., 2014). They could thus mainly reflect the activation of remote cortical and subcortical areas connected to the stimulation site.

A final limitation of our work lies in the way we defined the ROIs, i.e. simply by considering the regions around the targets used by the neuronavigation system. New optimized methods for modelling the electrical field induced by TMS (Thielscher et al., 2011) could be used in the future to define ROIs in a more accurate way according to excitation threshold. Furthermore, accurate biophysical modelling could also improve the tuning of stimulation intensity across sites that would then be based not only on scalp-cortex distances (Stokes et al., 2007; 2005) but also on the actual individual anatomy. 


\subsection{Functional cytoarchitectonics}

The mode analysis of LSA time series identified networks sharing intrinsic dynamical signatures in response to TMS single pulses: parietal-temporal, parietal-occipital, frontal, or motor. Parietaloccipital networks showed a resonant frequency in the alpha band, whereas the motor cortex mainly oscillated in the beta band. In healthy conditions, our methodology could be further developed as a functional cytoarchitectonic approach, that is a new non-invasive way to parcel the human superficial cerebral cortex on the basis of the dynamics of its local neuronal circuits' response. Parcellation of the cortex has a long history in neuroscience (Zilles and Amunts, 2010). The search for precise anatomical boundaries from local cytoarchitectural, myeloarchitectural, or receptoarchitectural features, is motivated by the assumption that structure determines function, which is essentially verified for primary areas (Cloutman and Lambon Ralph, 2012). For higher cognitive functions, computational neuroanatomy also relies upon the definition of hierarchies based on interlaminar patterns of long range connections to define distributed cortical hierarchies (Cloutman and Lambon Ralph, 2012; Crick and Koch, 1998; Hilgetag and Grant, 2010). Using neural mass models, it can be shown evoked EEG responses within cortical hierarchies directly depend upon the laminar pattern of connectivity (David et al., 2005). Inferences on intrinsic neuronal dynamics can thus be used to go beyond pure structural parcellation towards an anatomofunctional one, the main assumption being that neural communication is facilitated between neuronal populations showing similar resonant frequencies, or modes (Fries, 2005). Nowadays, non-invasive functional parcellation methods essentially rely on MRI techniques, such as restingstate functional MRI (Song et al., 2014; Yeo et al., 2011) and diffusion MRI tractography (Cloutman and Lambon Ralph, 2012; Tang et al., 2014). These methods are able to parcel a defined brain area in respect to a connectivity score, using both functional and/or anatomical connectivity. 
However, there is only few similar approaches based on electrophysiological recordings, although it has been proved that some of its characteristics are strongly modulated by topological and cytoarchitectural features, including the density of pyramidal cells (Fernández-Ruiz et al., 2013; Kajikawa and Schroeder, 2011; Murakami and Okada, 2006). Several studies using direct cortical electrical stimulation also showed that the properties of the neuronal responses depend a lot on the intrinsic cytoarchitecture or connectivity patterns of the stimulated area, in both animals (Luppino et al., 1991) and humans (Keller et al., 2014).

We propose that combining TMS and EEG can be used to develop functional cytoarchitectonics with the ultimate goal to parcel the human brain on the basis of distributed neuronal populations sharing common dynamical properties of their impulse response. TEP may indeed contain sufficient information to make inferences on local anatomical differences, because different interconnections and proportions of pyramidal cells, inhibitory and excitatory interneurons, automatically lead to differences in the macroscopic electrophysiogical responses to local electrical currents. For example, our results support the fact that the primary motor cortex has a very specific anatomical organization, which reacts in a unique manner to the stimulation, potentially due to the absence of cortical layer IV and to the presence of the large cortico-spinal pyramidal neurons. Furthermore, the pyramidal neurons of layer $\mathrm{V}$ are particularly large in the motor/premotor areas and in the median part of the occipital lobe (van den Heuvel et al., 2015), where we also observed the greatest early LSA (from 15 to $45 \mathrm{~ms}$, see Fig. 4b). Another interesting example is to note that dynamical properties of LSA were shared between the superior occipital lobe and the parietal lobe, or between the parietal lobe and the frontal lobe. It suggests the presence of distributed and overlapping functional networks resonating in different modes. However, the spatial resolution of our data is rather coarse because of the limited number of cortical targets and of EEG electrodes. Further 
studies are needed to go beyond our results, and in particular to challenge the spatial precision of the segregation that could achieve such a method based on a noninvasive neurophysiological approach. Moreover, the direct relation between our parcellation results and human cytoarchitectonics features is yet to be established, by correlating our data with extensive cytoarchitectonic databases.

Despite the limited spatial precision, the networks emerging from our study highly correlate with the resting state network (RSN) already described from low frequency signal fluctuations in fMRI (Song et al., 2014; Yeo et al., 2011) and more recently from phase-amplitude coupling synchronization between low (theta - alpha) and high (gamma) frequencies in magnetoencephalography (Florin and Baillet, 2015). Both techniques revealed that the spontaneous brain activity can be segregated into different RSN, ranging from local sensory-motor networks to large scale ones involving associated areas. These findings correlate with our results, regarding the spatial clusterization of LSA. Here using an active probing approach, we further showed that those networks share some common intrinsic frequency signatures, mainly composed of alpha and low gamma bands. Since RSN segregation in magnetoencephalography is based on phase-amplitude coupling between alpha and high gamma bands (Florin and Baillet, 2015), our new approach has the potential to map precisely the spectral properties of cortical areas supporting cross-frequency communication for large-scale neural communication.

\subsection{Potential applications}

The analysis of LSA modes and their related oscillatory contents can be turned into the identification of biomarkers of the integrity of cortical networks. A recent study supports this idea, 
by showing that the resonant frequency of the premotor area could be modified in several psychiatric diseases, like major depressive disorders or schizophrenia (Canali et al., 2015; Ferrarelli et al., 2012). EEG responses to TMS are also mental state dependent, as recently reported by a study showing that the resonant frequency of the occipital area was modulated by visual attention (Herring et al., 2015). Investigating mental state influence on LSA modes could give new insights regarding the variance of TMS (and repetitive TMS) aftereffects across subjects (Beynel et al., 2014). Other applications are the monitoring of stroke patient rehabilitation, since LSA directly reflects the integrity of cortical networks, or the differential diagnosis between vegetative and minimally conscious states. It has been shown that some of the characteristics of TEPs over the primary motor cortex, the superior frontal and parietal gyrus contain crucial information for the diagnosis of disorders of consciousness (Casali et al., 2013; Ragazzoni et al., 2013; Rosanova et al., 2012). Extending these findings towards the whole cortex could be valuable. Finally, the potential ability of this methodology in studying resting state networks could give new insights regarding its modulation by neurodegenerative diseases (Baggio et al., 2015; Spetsieris et al., 2015).

Overall, identifying intrinsic dynamical properties of cortical microcircuits opens many avenues for modelling both segregation and integration of neural information, with many potential applications in brain physiology and pathophysiology.

\section{Authors contribution}

S.H., C.M., A.C. and O.D. designed research. S.H., T.B. and L.B. performed research. S.H., T.B. and O.D. analysed data. SH, C.M., A.C. and O.D. wrote the paper. 


\section{Acknowledgements}

This work was funded by the Pôle Grenoble Cognition and the Agence Nationale pour la Recherche grant “ANR-15-CE37-0015-01”. Data were acquired on a platform of France Life Imaging network partly funded by the grant "ANR-11-INBS-0006". The authors declare no conflict of interest. 


\section{References}

Awiszus, F., 2003. TMS and threshold hunting. Supplements to Clinical Neurophysiology 56, 1323.

Baggio, H.C., Segura, B., Junque, C., 2015. Resting-state functional brain networks in Parkinson's disease. CNS Neurosci Ther 21, 793-801. doi:10.1111/cns.12417

Bell, A.J., Sejnowski, T.J., 1995. An information-maximization approach to blind separation and blind deconvolution. Neural Comput 7, 1129-1159.

Beynel, L., Chauvin, A., Guyader, N., Harquel, S., Marendaz, C., 2014. Age-related changes in intracortical inhibition are mental-cognitive state-dependent. Biol Psychol 101, 9-12. doi:10.1016/j.biopsycho.2014.05.011

Blair, R.C., Karniski, W., 1993. An alternative method for significance testing of waveform difference potentials. Psychophysiology 30, 518-524.

Bortoletto, M., Veniero, D., Thut, G., Miniussi, C., 2015. The contribution of TMS-EEG coregistration in the exploration of the human cortical connectome. Neurosci Biobehav Rev 49, 114-124. doi:10.1016/j.neubiorev.2014.12.014

Bouyer, J.J., Montaron, M.F., Vahnée, J.M., Albert, M.P., Rougeul, A., 1987. Anatomical localization of cortical beta rhythms in cat. Neuroscience 22, 863-869.

Braack, ter, E.M., de Vos, C.C., van Putten, M.J.A.M., 2015. Masking the Auditory Evoked Potential in TMS-EEG: A Comparison of Various Methods. Brain Topogr 28, 520-528. doi:10.1007/s10548-013-0312-z

Brodmann, K., 1909. Vergleichende Lokalisationslehre der Grosshirnrinde. Johann Ambrosius Barth, Leipzig.

Calhoun, V.D., Liu, J., Adali, T., 2009. A review of group ICA for fMRI data and ICA for joint inference of imaging, genetic, and ERP data. NeuroImage 45, S163-72. doi:10.1016/j.neuroimage.2008.10.057

Canali, P., Sarasso, S., Rosanova, M., Casarotto, S., Sferrazza-Papa, G., Gosseries, O., Fecchio, M., Massimini, M., Mariotti, M., Cavallaro, R., Smeraldi, E., Colombo, C., Benedetti, F., 2015. Shared reduction of oscillatory natural frequencies in bipolar disorder, major depressive disorder and schizophrenia. J Affect Disord 184, 111-115. doi:10.1016/j.jad.2015.05.043

Carota, F., Posada, A., Harquel, S., Delpuech, C., Bertrand, O., Sirigu, A., 2010. Neural dynamics of the intention to speak. Cerebral Cortex 20, 1891-1897. doi:10.1093/cercor/bhp255 
Casali, A.G., Gosseries, O., Rosanova, M., Boly, M., Sarasso, S., Casali, K.R., Casarotto, S., Bruno, M.-A., Laureys, S., Tononi, G., Massimini, M., 2013. A theoretically based index of consciousness independent of sensory processing and behavior. Sci Transl Med 5, 198ra105. doi:10.1126/scitranslmed.3006294

Casarotto, S., Romero Lauro, L.J., Bellina, V., Casali, A.G., Rosanova, M., Pigorini, A., Defendi, S., Mariotti, M., Massimini, M., 2010. EEG responses to TMS are sensitive to changes in the perturbation parameters and repeatable over time. PLoS ONE 5, e10281. doi:10.1371/journal.pone.0010281

Chatfield, M., Mander, A., 2009. The Skillings-Mack test (Friedman test when there are missing data). Stata J 9, 299-305. doi:10.1002/hbm.20781

Chung, S.W., Rogasch, N.C., Hoy, K.E., Fitzgerald, P.B., 2015. Measuring Brain Stimulation Induced Changes in Cortical Properties Using TMS-EEG. Brain Stimulation. doi:10.1016/j.brs.2015.07.029

Cloutman, L.L., Lambon Ralph, M.A., 2012. Connectivity-based structural and functional parcellation of the human cortex using diffusion imaging and tractography. Front. Neuroanat. 6. doi:10.3389/fnana.2012.00034

Cona, F., Zavaglia, M., Massimini, M., Rosanova, M., Ursino, M., 2011. A neural mass model of interconnected regions simulates rhythm propagation observed via TMS-EEG. NeuroImage 57, 1045-1058. doi:10.1016/j.neuroimage.2011.05.007

Crick, F., Koch, C., 1998. Constraints on cortical and thalamic projections: the no-strong-loops hypothesis. Nature 391, 245-250. doi:10.1038/34584

David, O., Harrison, L., Friston, K.J., 2005. Modelling event-related responses in the brain. NeuroImage 25, 756-770. doi:10.1016/j.neuroimage.2004.12.030

Economo, Von, C.F., Koskinas, G.N., 1925. Die Cytoarchitektonik der Hirnrinde des erwachsenen Menschen. Springer, Berlin.

Fernández-Ruiz, A., Muñoz, S., Sancho, M., Makarova, J., Makarov, V.A., Herreras, O., 2013. Cytoarchitectonic and dynamic origins of giant positive local field potentials in the dentate gyrus. Journal of Neuroscience 33, 15518-15532. doi:10.1523/JNEUROSCI.0338-13.2013

Ferrarelli, F., Sarasso, S., Guller, Y., Riedner, B.A., Peterson, M.J., Bellesi, M., Massimini, M., Postle, B.R., Tononi, G., 2012. Reduced natural oscillatory frequency of frontal thalamocortical circuits in schizophrenia. Archives of general psychiatry 69, 766-774. doi:10.1001/archgenpsychiatry.2012.147

Florin, E., Baillet, S., 2015. The brain's resting-state activity is shaped by synchronized cross- 
frequency coupling of neural oscillations. NeuroImage 111, 26-35. doi:10.1016/j.neuroimage.2015.01.054

Fries, P., 2005. A mechanism for cognitive dynamics: neuronal communication through neuronal coherence. Trends Cogn Sci (Regul Ed) 9, 474-480. doi:10.1016/j.tics.2005.08.011

Fuggetta, G., Fiaschi, A., Manganotti, P., 2005. Modulation of cortical oscillatory activities induced by varying single-pulse transcranial magnetic stimulation intensity over the left primary motor area: a combined EEG and TMS study. NeuroImage 27, 896-908. doi:10.1016/j.neuroimage.2005.05.013

Ginhoux, R., Renaud, P., Zorn, L., Goffin, L., Bayle, B., Foucher, J., Lamy, J., Armspach, J.P., de Mathelin, M., 2013. A custom robot for Transcranial Magnetic Stimulation: first assessment on healthy subjects. Conf Proc IEEE Eng Med Biol Soc 2013, 5352-5355. doi:10.1109/EMBC.2013.6610758

Gosseries, O., Sarasso, S., Casarotto, S., Boly, M., Schnakers, C., Napolitani, M., Bruno, M.-A., Ledoux, D., Tshibanda, J.-F., Massimini, M., Laureys, S., Rosanova, M., 2015. On the cerebral origin of EEG responses to TMS: insights from severe cortical lesions. Brain Stimulation 8, 142-149. doi:10.1016/j.brs.2014.10.008

Gramfort, A., Papadopoulo, T., Olivi, E., Clerc, M., 2010. OpenMEEG: opensource software for quasistatic bioelectromagnetics. Biomed Eng Online 9, 45. doi:10.1186/1475-925X-9-45

Hegerl, U., Juckel, G., 1993. Intensity dependence of auditory evoked potentials as an indicator of central serotonergic neurotransmission: a new hypothesis. Biol Psychiatry 33, 173-187.

Herring, J.D., Thut, G., Jensen, O., Bergmann, T.O., 2015. Attention Modulates TMS-Locked Alpha Oscillations in the Visual Cortex. Journal of Neuroscience 35, 14435-14447. doi:10.1523/JNEUROSCI.1833-15.2015

Hilgetag, C.C., Grant, S., 2010. Cytoarchitectural differences are a key determinant of laminar projection origins in the visual cortex. NeuroImage 51, 1006-1017. doi:10.1016/j.neuroimage.2010.03.006

Ilmoniemi, R.J., Virtanen, J., Ruohonen, J., Karhu, J., Aronen, H.J., Näätänen, R., Katila, T., 1997. Neuronal responses to magnetic stimulation reveal cortical reactivity and connectivity. Neuroreport 8, 3537-3540.

Janssen, A.M., Oostendorp, T.F., Stegeman, D.F., 2015. The coil orientation dependency of the electric field induced by TMS for M1 and other brain areas. J NeuroEngineering Rehabil 12, 47. doi:10.1186/s12984-015-0036-2

Jones, E.G., 2000. Microcolumns in the cerebral cortex. Proc Natl Acad Sci USA 97, 5019-5021. 
Kajikawa, Y., Schroeder, C.E., 2011. How local is the local field potential? Neuron 72, 847-858. doi:10.1016/j.neuron.2011.09.029

Keller, C.J., Honey, C.J., Mégevand, P., Entz, L., Ulbert, I., Mehta, A.D., 2014. Mapping human brain networks with cortico-cortical evoked potentials. Philos Trans R Soc Lond, B, Biol Sci 369. doi:10.1098/rstb.2013.0528

Lioumis, P., Kičić, D., Savolainen, P., Mäkelä, J.P., Kahkonen, S., 2009. Reproducibility of TMSEvoked EEG responses. Hum Brain Mapp 30, 1387-1396. doi:10.1002/hbm.20608

Löfberg, O., Julkunen, P., Tiihonen, P., Pääkkönen, A., Karhu, J., 2013. Repetition suppression in the cortical motor and auditory systems resemble each other--a combined TMS and evoked potential study. Neuroscience 243, 40-45. doi:10.1016/j.neuroscience.2013.03.060

Luppino, G., Matelli, M., Camarda, R.M., Gallese, V., Rizzolatti, G., 1991. Multiple representations of body movements in mesial area 6 and the adjacent cingulate cortex: an intracortical microstimulation study in the macaque monkey. J Comp Neurol 311, 463-482. doi:10.1002/cne.903110403

Makeig, S., Bell, A.J., Jung, T.P., Sejnowski, T.J., 1996. Independent component analysis of electroencephalographic data, in: Touretzky, D., Mozer, M., Hasselmo, M. (Eds.), Advances in Neural Information Processing Systems. Advances in Neural Information Processing Systems, MIT Press Cambridge MA, p. 145:151.

Mattavelli, G., Rosanova, M., Casali, A.G., Papagno, C., Romero Lauro, L.J., 2013. Top-down interference and cortical responsiveness in face processing: a TMS-EEG study. NeuroImage 76, 24-32. doi:10.1016/j.neuroimage.2013.03.020

Miniussi, C., Thut, G., 2010. Combining TMS and EEG offers new prospects in cognitive neuroscience. Brain Topogr 22, 249-256. doi:10.1007/s10548-009-0083-8

Mueller, J.K., Grigsby, E.M., Prevosto, V., Petraglia, F.W., Rao, H., Deng, Z.-D., Peterchev, A.V., Sommer, M.A., Egner, T., Platt, M.L., Grill, W.M., 2014. Simultaneous transcranial magnetic stimulation and single-neuron recording in alert non-human primates. Nat Neurosci 17, 11301136. doi:10.1038/nn.3751

Murakami, S., Okada, Y., 2006. Contributions of principal neocortical neurons to magnetoencephalography and electroencephalography signals. J Physiol (Lond) 575, 925-936. doi:10.1113/jphysiol.2006.105379

Oostenveld, R., Fries, P., Maris, E., Schoffelen, J.-M., 2011. FieldTrip: Open source software for advanced analysis of MEG, EEG, and invasive electrophysiological data. Computational Intelligence and Neuroscience 2011, 156869. doi:10.1155/2011/156869 
Picazio, S., Veniero, D., Ponzo, V., Caltagirone, C., Gross, J., Thut, G., Koch, G., 2014. Prefrontal control over motor cortex cycles at beta frequency during movement inhibition. Curr Biol 24, 2940-2945. doi:10.1016/j.cub.2014.10.043

Ragazzoni, A., Pirulli, C., Veniero, D., Feurra, M., Cincotta, M., Giovannelli, F., Chiaramonti, R., Lino, M., Rossi, S., Miniussi, C., 2013. Vegetative versus minimally conscious states: a study using TMS-EEG, sensory and event-related potentials. PLoS ONE 8, e57069. doi:10.1371/journal.pone.0057069

Reuter, M., Schmansky, N.J., Rosas, H.D., Fischl, B., 2012. Within-subject template estimation for unbiased longitudinal image analysis. NeuroImage 61, 1402-1418. doi:10.1016/j.neuroimage.2012.02.084

Rogasch, N.C., Fitzgerald, P.B., 2013. Assessing cortical network properties using TMS-EEG. Hum Brain Mapp 34, 1652-1669. doi:10.1002/hbm.22016

Rogasch, N.C., Thomson, R.H., Farzan, F., Fitzgibbon, B.M., Bailey, N.W., Hernandez-Pavon, J.C., Daskalakis, Z.J., Fitzgerald, P.B., 2014. Removing artefacts from TMS-EEG recordings using independent component analysis: Importance for assessing prefrontal and motor cortex network properties. NeuroImage 101, 425-439. doi:10.1016/j.neuroimage.2014.07.037

Rosanova, M., Casali, A., Bellina, V., Resta, F., Mariotti, M., Massimini, M., 2009. Natural frequencies of human corticothalamic circuits. Journal of Neuroscience 29, 7679-7685. doi:10.1523/JNEUROSCI.0445-09.2009

Rosanova, M., Gosseries, O., Casarotto, S., Boly, M., Casali, A.G., Bruno, M.-A., Mariotti, M., Boveroux, P., Tononi, G., Laureys, S., Massimini, M., 2012. Recovery of cortical effective connectivity and recovery of consciousness in vegetative patients. Brain 135, 1308-1320. doi:10.1093/brain/awr340

Roy, D., Sigala, R., Breakspear, M., McIntosh, A.R., Jirsa, V.K., Deco, G., Ritter, P., 2014. Using the virtual brain to reveal the role of oscillations and plasticity in shaping brain's dynamical landscape. Brain Connect 4, 791-811. doi:10.1089/brain.2014.0252

Salkoff, D.B., Zagha, E., Yüzgeç, Ö., McCormick, D.A., 2015. Synaptic Mechanisms of Tight Spike Synchrony at Gamma Frequency in Cerebral Cortex. Journal of Neuroscience 35, 1023610251. doi:10.1523/JNEUROSCI.0828-15.2015

Song, X., Zhang, Y., Liu, Y., 2014. Frequency specificity of regional homogeneity in the restingstate human brain. PLoS ONE 9, e86818. doi:10.1371/journal.pone.0086818

Spetsieris, P.G., Ko, J.H., Tang, C.C., Nazem, A., Sako, W., Peng, S., Ma, Y., Dhawan, V., Eidelberg, D., 2015. Metabolic resting-state brain networks in health and disease. Proc Natl 
Acad Sci USA 112, 2563-2568. doi:10.1073/pnas.1411011112

Stokes, M.G., Chambers, C.D., Gould, I.C., English, T., Mcnaught, E., McDonald, O., Mattingley, J.B., 2007. Distance-adjusted motor threshold for transcranial magnetic stimulation. Clin Neurophysiol 118, 1617-1625. doi:10.1016/j.clinph.2007.04.004

Stokes, M.G., Chambers, C.D., Gould, I.C., Henderson, T.R., Janko, N.E., Allen, N.B., Mattingley, J.B., 2005. Simple metric for scaling motor threshold based on scalp-cortex distance: application to studies using transcranial magnetic stimulation. J Neurophysiol 94, 4520-4527. doi:10.1152/jn.00067.2005

Tadel, F., Baillet, S., Mosher, J.C., Pantazis, D., Leahy, R.M., 2011. Brainstorm: a user-friendly application for MEG/EEG analysis. Computational Intelligence and Neuroscience 2011, 879716. doi:10.1155/2011/879716

Tang, X., Yoshida, S., Hsu, J., Huisman, T.A.G.M., Faria, A.V., Oishi, K., Kutten, K., Poretti, A., Li, Y., Miller, M.I., Mori, S., 2014. Multi-contrast multi-atlas parcellation of diffusion tensor imaging of the human brain. PLoS ONE 9, e96985. doi:10.1371/journal.pone.0096985

Thielscher, A., Opitz, A., Windhoff, M., 2011. Impact of the gyral geometry on the electric field induced by transcranial magnetic stimulation. NeuroImage 54, 234-243. doi:10.1016/j.neuroimage.2010.07.061

Thut, G., Veniero, D., Romei, V., Miniussi, C., Schyns, P., Gross, J., 2011. Rhythmic TMS causes local entrainment of natural oscillatory signatures. Curr Biol 21, 1176-1185. doi:10.1016/j.cub.2011.05.049

van de Ruit, M., Perenboom, M.J.L., Grey, M.J., 2015. TMS brain mapping in less than two minutes. Brain Stimulation 8, 231-239. doi:10.1016/j.brs.2014.10.020

van den Heuvel, M.P., Scholtens, L.H., Feldman Barrett, L., Hilgetag, C.C., de Reus, M.A., 2015. Bridging Cytoarchitectonics and Connectomics in Human Cerebral Cortex. Journal of Neuroscience 35, 13943-13948. doi:10.1523/JNEUROSCI.2630-15.2015

Vernet, M., Brem, A.-K., Farzan, F., Pascual-Leone, A., 2015. Synchronous and opposite roles of the parietal and prefrontal cortices in bistable perception: a double-coil TMS-EEG study. Cortex; a journal devoted to the study of the nervous system and behavior 64, 78-88. doi:10.1016/j.cortex.2014.09.021

Wassermann, E., Epstein, C., Ziemann, U., 2012. The Oxford handbook of transcranial stimulation, oxfordhandbooks.com. Oxford University Press. doi:10.1093/oxfordhb/9780198568926.001.0001

Yeo, B.T.T., Krienen, F.M., Sepulcre, J., Sabuncu, M.R., Lashkari, D., Hollinshead, M., Roffman, 
J.L., Smoller, J.W., Zöllei, L., Polimeni, J.R., Fischl, B., Liu, H., Buckner, R.L., 2011. The organization of the human cerebral cortex estimated by intrinsic functional connectivity. J Neurophysiol 106, 1125-1165. doi:10.1152/jn.00338.2011

Zilles, K., Amunts, K., 2010. Centenary of Brodmann's map - conception and fate. Nat Rev Neurosci 11, 139-145. doi:10.1038/nrn2776 